\title{
A remote-controlled device for long-term blood collection from freely moving, socially housed animals
}

\author{
KATHY L. BENTSON, FRANK P. MILES, CLIFFORD A. ASTLEY, and ORVILLE A. SMITH \\ Regional Primate Research Center, University of Washington, Seattle, Washington
}

\begin{abstract}
A remote-controlled device was developed for injection of fluids and collection of blood samples from freely moving, socially housed animals via an indwelling catheter. Samples are collected at times chosen by the investigator, and the animal is not aware that sampling is occurring. This technique allows measurement of plasma catecholamines and other substances that fluctuate rapidly, are affected by psychosocial stimuli, and are altered by capture and other stressful activities that often accompany sample collection. Rapid retrieval of samples is facilitated by remote-controlled injection of an anesthetic. The device has been used to collect blood from dominant and subordinate male baboons before and during events such as chases and presentation of food treats. Heart rate and blood pressure were measured via radio telemetry, and behavior was recorded on videotapes. This technique allowed construction of multifaceted physiological profiles of social roles and behaviors.
\end{abstract}

Levels of some blood-borne substances change quickly during social behavior and physical activity. Electrolytes and blood gases, for example, change rapidly and continuously during exercise. It is not possible to collect blood or inject an agent by conventional means without interrupting such activities. In some situations, it is possible to obtain samples using venipuncture or to inject a substance via needle and syringe, but the process may introduce variables that compromise the validity of the measures that are obtained. The stressful events that precede and accompany sample collection, such as removal from the home environment, interactions with humans, immobilization, exposure to a needle, and sedation, can affect the levels of many blood-borne substances, including plasma catecholamines, the hormones of the en-

This research was supported by Grants RR00166 and HL50351 from the National Institutes of Health, MH 49033 from the National Institutes of Mental Health, and by a grant from the University of Washington Graduate School Fund. Much of the information in this manuscript is contained within a doctoral dissertation written by K.L.B. Results arising from use of the device have been published in abstracts that are listed in the references and at greater length in the dissertation. The authors gratefully acknowledge the Bioengineering Division at the Washington Regional Primate Research Center for invaluable assistance, Courtney Holmes for plasma catecholamine assays using techniques developed in David Goldstein's laboratory at NIH, Marj Domenowske for illustrations, Kate Elias for editorial assistance, and John Capitanio for assistance in preparing the manuscript. Correspondence and queries about the blood collection/fluid injection unit should be addressed to K. L. Bentson, now at the California Regional Primate Research Center, 1 Shields Ave., University of California, Davis, CA 95616 (e-mail: klbentson@ucdavis.edu).Correspondence about radio telemetry and construction of the controller card and command link should be addressed to F. P. Miles, Bioengineering Department, Washington Regional Primate Research Center, HSB Box 357330 , University of Washington, Seattle, WA 98195 (e-mail: frankm(@),bart.rprc.washington.edu) tire adrenocortical and reproductive axes, endorphins, thyroid hormones, prolactin, growth hormone, glucose, insulin, glucagon, and various cytokines.

The best way to gather valid experimental data about variables that would be affected by procedure-related stress is to obtain blood and inject agents while subjects carry on their regular routines and are not aware of the investigator's activities. One method is the use of a "tethering" technique in which a chronically implanted catheter extends from the subject to a location out of the animal's sight (Morton, Knitter, Smith, Susor, \& Schmitt, 1987). Although this technique does provide useful information, it has limitations. Because the catheter must be protected from damage by animals or objects in the environment, the animal typically must be caged alone and has a limited range of movement.

Another method is the use of remote-controlled blood collection devices. Such devices have been used to sample blood from greyhounds during races (Schmalzried, Toll, Devore, \& Fedde, 1992), seals during dives (Hill, 1986), unrestrained birds at rest (Scheid \& Slama, 1975), and rhesus monkeys during sexual activity (Herndon, Perachio, Turner, \& Collins, 1981). They have made it possible to measure blood-borne substances whose levels change quickly during events, and they have eliminated some obstacles to obtaining blood without influencing levels of substances through the process of sample collection. However, devices used to collect samples during events typically are designed for short-term use and are attached on the day of sample collection. This involves interactions with humans, a departure from the normal routine, the introduction of a novel piece of equipment, and, in some cases, minor medical procedures or sedation.

We have developed an improved remote-controlled blood sampling and fluid injection device that can be 
worn by animals for long-term studies. The animals become familiar with the pack used to carry the device for at least 2 weeks before data collection begins, and use of the device does not require deviations from the animals' routines prior to sample collection. The device allows the collection of heparin-free samples from subjects as they move freely about a large area and interact with other animals in their home environment. Up to three samples can be obtained per session from each subject at times of the investigator's choosing. Blood in the catheter is replaced with heparin injected by remote control, which gives the investigator the option of collecting samples some minutes apart without running a risk of clot formation. The subjects are not aware that blood is being collected. After sample collection, the animals can be sedated by remote control so that samples can be retrieved immediately, thereby increasing the accuracy in measurement of blood-borne substances that degrade at room temperature.

This remote-controlled device has been used in combination with telemetered cardiovascular information (Spelman et al., 1991) and videotapes of behavior (Astley et al., 1991) to measure plasma catecholamines and cortisol in dominant and subordinate baboons. Samples were collected during events such as anticipation and presentation of preferred food and noncontact exposure to a large baboon that was not a member of the group (Bentson, 1998; Bentson et al., 1995, 1996, 1997).

\section{DESCRIPTION OF THE DEVICE}

The remote-controlled blood sampling device, shown schematically in Figure 1, has three main components: a controller card, which specifies the parameters of operation, interprets and acts on signals sent by remote control, and activates the transmitter; a command link, which sends commands, such as "turn on the transmitter" or "open valve 2 for 30 sec" (i.e., collect a blood sample); and a collection/fluid injection unit, which collects blood samples and injects fluids through the catheter. The entire system is powered by three AA lithium-sulfur primary cells (Energy Sales, Redmond, WA). Physiological data and information about functions of the device are sent via radio telemetry to a recording booth adjacent to the compound. The telemetry equipment has been described previously (Spelman et al., 1991). The flow of information to and from the animal's backpack is illustrated in Figure 2. The pack is made of a sturdy but lightweight plastic (Kydex) and lined with foam that fits the contours of the animal's back. A harness is used to hold the pack in place and allow the fit of the pack to be tailored to each animal. Subjects and other animals in the compound quickly determine they cannot remove the pack, and attempts to do so have not been observed to persist. The controller card and collection/injection unit are placed in a backpack, which, along with the harness and equipment, weighs $1.5 \mathrm{~kg}$. This can be worn comfortably by animals the size of adult male baboons ( $16-35 \mathrm{~kg}$ ). A smaller device that allows collection of two samples per session is being developed for use with male rhesus monkeys that weigh as little as $10 \mathrm{~kg}$.

\section{Controller Card}

The controller card is the heart of the system. Measuring $10 \times 5.6 \times 1.5 \mathrm{~cm}$, the four-layer circuit board contains a microprocessor (Motorola 68HC11E2), a transmitter, and connecting pins for the valve leads of the collection/injection unit. The microprocessor includes an 8-bit analog-to-digital converter, timer array, parallel digital input/output, and asynchronous ports for communication and control. The transmitter is a lowpower crystal-controlled narrowband FM transmitter. Transmitters on different controller cards have different frequencies so that data can be collected from more than one animal in the same enclosure. The transmitting antenna is a simple length of wire wrapped around one of the straps holding the backpack. Components are surfacemounted on both sides of the circuit board, thereby minimizing the size and weight of the controller card. The card is enclosed in a lightweight protective case that is open along one edge to allow connection to the valves, pressure transducer, and batteries.

\section{Command Link}

The command link relays information between the experimenter and the backpack. Essentially, this component consists of a signaling device, infrared light-emitting diodes (LEDs), an infrared receiver, and a serial decoder. The signaling device resembles a television remote control unit and is held by the investigator. The LEDs are located in the ceiling of the animal compound and in the signaling device. Signals are received by an infrared receiver on the pack and relayed to the serial decoder that is part of the controller card. Each animal's controller card is programmed with a unique "selector address." The pack also has two red LEDs visible to its exterior. One turns on transiently when an infrared signal is received; the other stays on as long as a valve is open. This provides visual feedback to the investigator that a signal was received and the command is being processed. When the investigator presses a control button on the signaling device, the room is flooded with infrared light pulsing at $40 \mathrm{kHz}$. The light is modulated by a digital code string, which depends on the button pressed and the chosen animal's selector address. The infrared receiver in the backpack responds to these pulses. When the serial decoder matches the code string selector address with the controller card address, a 4-bit control value is latched. This interrupts or restarts the microprocessor and causes a red LED on the exterior of the backpack to flash, letting the experimenter know that a valid code was received. The infrared LEDs in the signaling device allow signals to be sent when the animals are not in the compound (e.g., when they are in an adjacent holding area). The signaling device directs commands to one animal at 


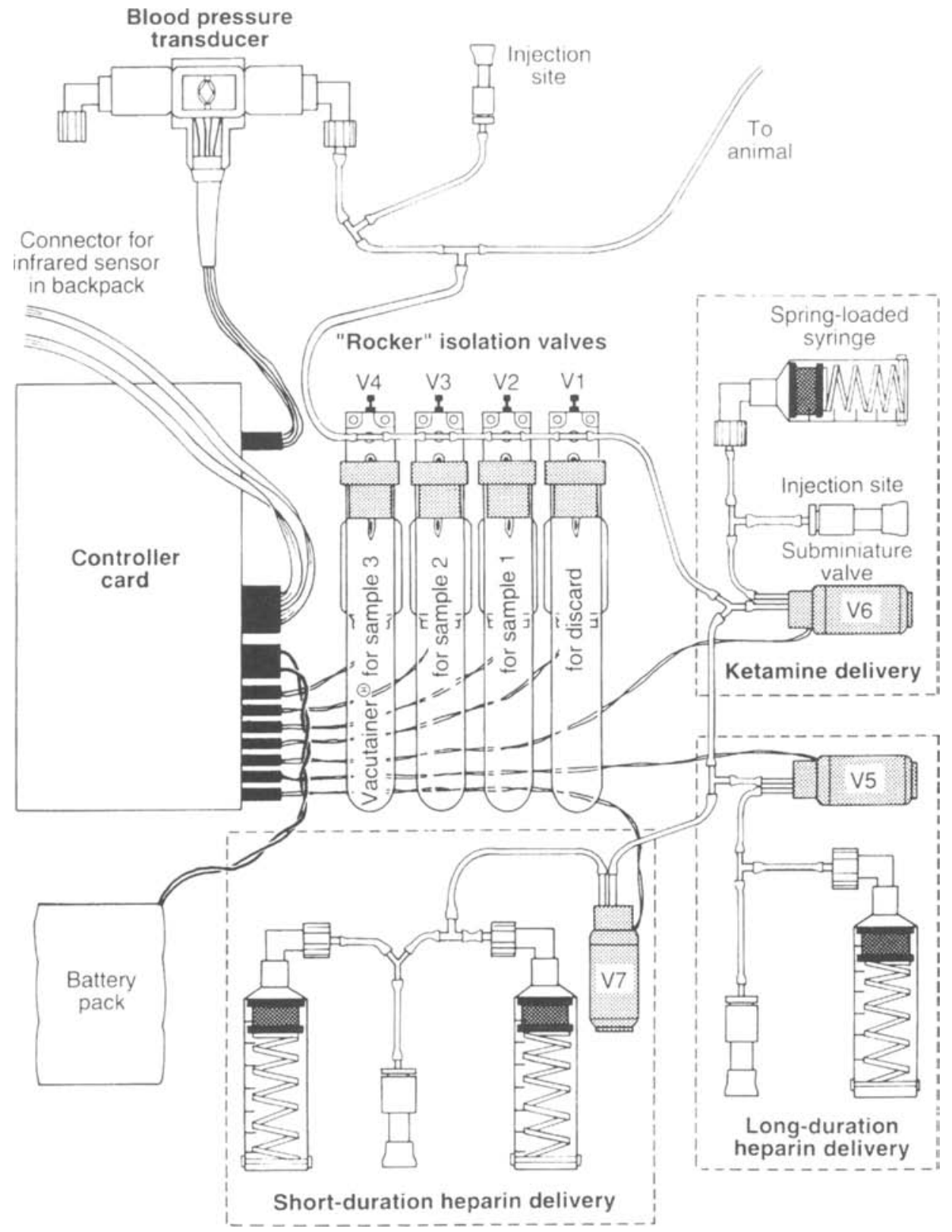

Figure 1. Remote-controlled blood sampling device and blood pressure transducer. These components, contained within a backpack, allow measurement of heart rate and blood pressure via radio telemetry, collection of blood by remote control, and delivery of fluids through the catheter. The controller card processes infrared signals sent by the investigator. Rocker valves are used to clear heparin from the catheter and draw blood into collection tubes. Subminiature valves and spring-loaded syringes are used to release fluids into the catheter.

a time; the command can separately target up to 32 subjects in one enclosure. The compound in Seattle is $3.8 \mathrm{~m}$ wide $\times 7.0 \mathrm{~m}$ long, but the addition of more infrared LEDs would allow signals to be received in a larger enclosure.

\section{Collection/Injection Unit}

The collection/injection unit (Figure 1 and Table I) consists of valves, collection tubes, syringes, and a cath- eter. The valves control movement of fluids into and out of the catheter, which extends from the backpack to the animal's aorta. When a valve attached to a blood collection tube opens, the vacuum in the tube combines with the animal's arterial pressure to move heparin or blood into the tube (Schmalzried et al., 1992). When a valve attached to a syringe opens, a spring behind the plunger pushes fluid into the catheter (Spelman et al., 1991). Rocker isolation valves (Angar Scientific Co., Cedar 


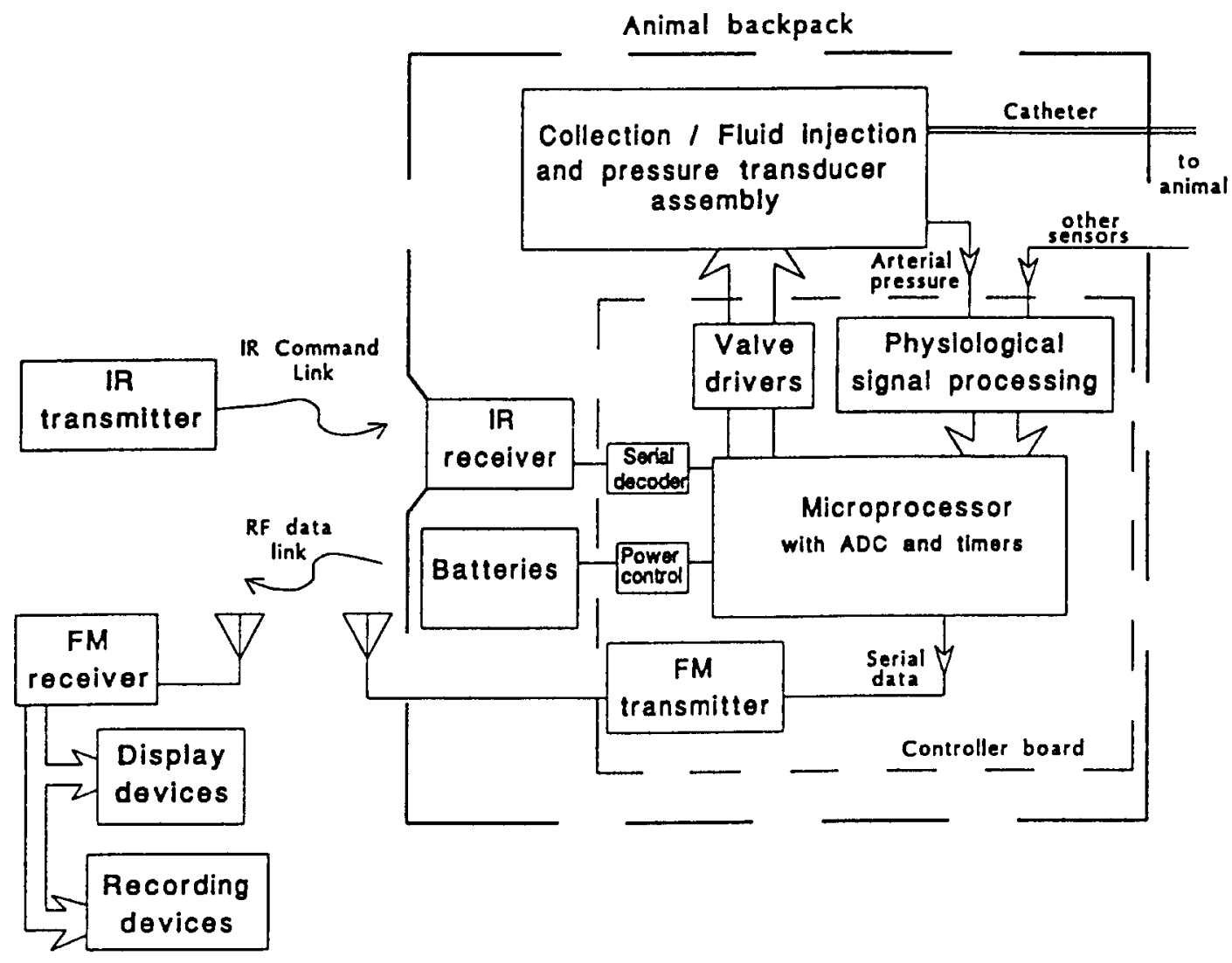

Figure 2. Contents of the backpack and flow of information to and from the pack components. Infrared (IR) signals send information from the investigator to the controller card. Physiological data and information about device function are sent to a nearby recording booth via radio telemetry.

Knolls, NJ; Model 45810061 ES) are used for blood collection, and subminiature valves (Angar Model 407611 modified to have 18-ga posts) are used for fluid injection. The controller card can accommodate up to eight valves. The components of the collection/injection unit are attached to each other, and the entire unit is connected to the catheter, with Tygon tubing. Connectors between segments of tubing are obtained from Value Plastics (Fort Collins, CO). The unit is encased in a lightweight metal box.

Each new rocker valve is tested when it arrives from the factory and, if necessary, adjusted to allow blood to flow through it at an adequate rate when in the open position while not allowing heparin to be pulled into the va- cutainer when the valve is closed. Few valves require adjustment. Tubing leads from the upper post of the valve to a T-shaped connector that puts the subunit in line with the rest of the device and with the catheter. The lower post is connected to a blood collection apparatus, which is newly constructed and tested before each blood draw. The apparatus includes a T-shaped connector with three pieces of tubing (Figure 3). One leads to a vacuum gauge during testing and is attached to the valve's lower post during actual use. The second leads to a 15-ga needle that has had the hub removed and is bent into a gentle curve and then to a $10-\mathrm{ml}$ blood collection tube (Vacutainer, Rutherford, NJ). Shrink-wrap tubing prevents vacuum loss from the intersection between the tubing

Table 1

Components of the Blood Collection/Fluid Injection Unit

\begin{tabular}{cll}
\hline Valve & \multicolumn{1}{c}{ Tube or Syringe Controlled } & \multicolumn{1}{c}{ Function } \\
\hline 1 & Blood collection tube for discard & Removes heparin from the catheter before samples are collected \\
2 & Blood collection tube for Sample 1 & Collects Blood Sample 1 \\
3 & Blood collection tube for Sample 2 & Collects Blood Sample 2 \\
4 & Blood collection tube for Sample 3 & Collects Blood Sample 3 \\
5 & Spring-loaded syringe for long-duration heparin delivery & Injects heparin to clear blood from the catheter after sample collection \\
6 & Spring-loaded syringe for sedation with ketamine or Telazol & Sedates the animal for retrieval of blood samples \\
7 & Spring-loaded syringe for short-duration heparin delivery & Injects <0.5 ml of heparin for routine catheter maintenance \\
\hline
\end{tabular}




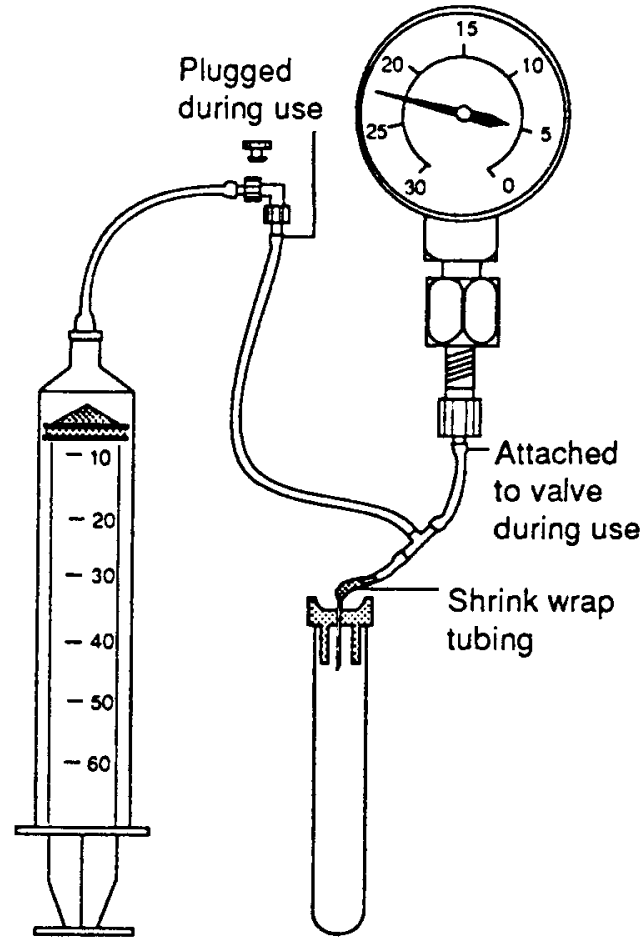

Figure 3. Process for creating a vacuum in the blood collection apparatus. A T-shaped connector has three pieces of tubing leading from it. One leads to a vacuum gauge during testing and is attached to a rocker valve during actual use. The second leads to a curved needle and then to a blood collection tube. Shrink-wrap tubing prevents vacuum loss from the intersection between the tubing and the barrel of the needle. The tubing leading off the third side of the "T" is used to apply a vacuum to the blood collection tube. Air is drawn out of the tube with a syringe that is temporarily attached to a luer at the tip of the tubing. After a vacuum is created, the syringe is detached and a plug is inserted in the luer.

and the barrel of the needle. The area of the Vacutainer stopper that is pierced by the needle is sealed with petroleum jelly. The tubing leading off the third side of the " $T$ " is used to apply a vacuum to the blood collection tube. Air is drawn out of the tube with a syringe that is temporarily attached to a luer at the tip of the tubing. The syringe is then detached, and a plug is inserted in the luer.

Spring-loaded syringes hold injection fluids and release their contents into the animal's catheter upon receiving instruction via the command link. The amount of fluid to be released is controlled by the length of time the valve remains open. Valve times are set with a resolution of $50 \mathrm{msec}$ for each individual valve before the unit is attached to the animal's catheter. The syringes, which are described elsewhere (Spelman et al., 1991), are made from shortened $10-\mathrm{ml}$ disposable plastic syringes and stainless steel springs, such as model LCM$110 \mathrm{GH}-7$ (Lee Spring, Irwindale, CA). The length of time before syringes start sticking is increased by never removing the plunger from the syringe body during con- struction. After a syringe starts to stick, it is possible to extend the useful life of the syringe by lubricating the body with a silastic lubricant. We preferred, though, to make new syringes when old ones began to stick.

\section{OPERATION OF THE DEVICE}

Before the device is placed in the backpack, the microprocessor on the controller card is computer-programmed to specify three parameters of operation:

1. The amount of time each valve is to stay open. Valves can be kept open as briefly as $50 \mathrm{msec}$ or as long as $40 \mathrm{sec}$. This time affects how much blood will be collected or how much fluid will be injected. The rocker valves that control sample collection are set to stay open long enough to maximize the amount of blood that can be collected regardless of variations in vacuum and arterial pressure.

2. The number of times the same infrared signal must be received by the microprocessor before the valve opens. Increasing the number reduces the chances that an incompletely received or garbled signal will be misinterpreted. We specify three identical receptions for all commands except the command to sedate the baboon, which requires seven identical receptions. In our experience, this redundancy completely prevents wrong interpretations of commands. It takes $189 \mathrm{msec}$ to send a signal seven times, so this safeguard is noticeable only on rare occasions when signal reception is poor.

3. The minimum length of time after a signal is processed before the same signal can be processed again. This setting eliminates unintentional repeated processing of short-duration executed commands that can result from holding down the button on the signaling device too long. For example, if the valve controlling heparin release for catheter maintenance is designated to be open only $0.4 \mathrm{sec}$ but the button is pressed longer than that, the signal continues to be sent. However, heparin is not released again because the microprocessor does not carry out commands for a period of time (e.g., $3 \mathrm{sec})$ after the original signal was processed.

During data collection, the controller card receives infrared signals sent by the investigator and performs two major functions. Its first function is to turn the transmitter on and off. Leaving the transmitter off when data are not being collected increases the life of the battery. When the transmitter is on, it sends blood pressure data from the pack to a receiver located in the recording booth. The transmitter is modulated by the asynchronous serial output. As soon as the serial buffer is empty, the next physiological data byte is loaded into it. Data transmission is at 4,800 baud. The receiver processes the blood pressure signal and produces a heart rate record (Spelman et al., 1991). The transmitter also identifies the last signal the controller card received and reads the battery voltage.

The second function of the controller card is to interpret and execute a variety of commands that control 
valve openings and closings. Signals can be sent to control each valve individually or as part of a sequence. For example, during a two-sample blood draw session, three commands may be used (see Table 1 and Figure 1). First, a command initiates a sequence in which Valve 1 opens for $4 \mathrm{sec}$ to allow heparin to be drawn into a blood collection tube for later discard so that a heparin-free blood sample can be obtained. Valve 1 closes, and Valve 2, which controls access to a different blood collection tube, opens for $30 \mathrm{sec}$ to collect the first sample. A red LED on the backpack and changes in the blood pressure record let the experimenter know when the valve sequence is completed. Second, a command is sent to open Valve 5 for 1 sec, which permits the injection of heparin to push blood out of the catheter. Third, when the investigator wants to collect the second sample, a signal is sent to open Valve 1 (again providing access to the "discard" blood collection tube for heparin clearance) and then to open Valve 3 to collect the second sample in yet another blood collection tube.

\section{TECHNICAL CONSIDERATIONS}

Several mechanical relationships must be observed for proper functioning of the unit. So that blood can be cleared from all tubing between blood collections, the sites of attachment for the valves used for blood collection must be closer to the catheter than the attachment sites for the valves that control fluid release. Similarly, the valve controlling access to the blood collection tube used to clear heparin from the catheter must be attached farther from the catheter than the attachment sites for the valves used to collect blood samples so that heparin can be removed from the tubing between the catheter and the blood collection valves.

The timing of the collection/injection valves may need occasional adjustments owing to long-term changes in catheter patency and mechanical degradation of the syringe body, springs, or other components. This may be done over a temporarily connected serial link cable between the controller board microprocessor and a host computer.

The entire unit must not leak under the force of constantly applied arterial pressure, because even a slow leak will cause blood to advance up the catheter, forming clots. Every surface of the device that shares fluid with the catheter must be accessible for sterilization. Finally, the unit must be able to hold the vacuum in the blood collection tubes from the time the device is put in the pack to the time samples are collected (typically 2 or 3 days, but sometimes a week). Tips for minimizing the occurrence of fluid leaks and maximizing the probability that the vacuum will hold are presented in the Discussion section.

The pressure transducer is calibrated at each battery replacement or reattachment to the controller card. The pressure signal is useful not only as an interesting physiological indicator but also as a test of valve actuation and catheter patency. Other physiological signals may be measured and transmitted to the experimenter: up to four "fast" physiological signals may each be transmitted at $\sim 100$ samples/sec. Each of these four signals has its own amplifier and antialias filter that precedes sampling and analog-to-digital conversion within the microprocessor. An additional three signals may be transmitted at a much slower rate, $\sim 1$ sample/sec. This is useful for slowly varying physiological information, such as body temperature, or for system information, such as battery voltage and reporting the last code received.

When not in use, all functions except for the infrared receiver and decoder may be turned off. This extends the time between battery replacements to about 10 days, assuming that the total "power on" time is limited, as it was in the present study, to $\sim 3.5 \mathrm{~h} /$ week. Under those conditions, more than $90 \%$ of power consumption is devoted to $24 \mathrm{~h}$ /day monitoring for incoming signals, so moderate increases in the "power on" time would not greatly reduce the life of the battery.

\section{USE OF THE SYSTEM}

Over a 2-year period, we used this device in several studies of freely moving, socially housed baboons. Before the studies began, the baboons were fitted with backpacks and acclimated to the packs for a week. A catheter was then surgically inserted via the axillary artery into the aorta; the peripheral end of the catheter exited from the animal's back and, within the pack, was attached to the blood collection device. No data were collected for a week after catheter surgery. The catheter was kept patent by remote-controlled release of $<0.5 \mathrm{ml}$ of heparin twice a day. On sample collection days, cardiovascular and behavioral data were recorded throughout the session. At designated time points, blood samples were collected by remote control. For example, during a typical food anticipation/presentation session, signaled by the ringing of a bell, samples were taken from 2 subjects at three time points: before the bell, when both animals had been sitting quietly for at least $2 \mathrm{~min} ; 2 \mathrm{~min}$ after the bell, while the baboons were watching the food being cut up; and $4 \mathrm{~min}$ after the bell, while the food was available in the compound. After the samples were collected, a door leading to a holding area was raised and the baboons entered the holding area, where they were sedated by remote control. The blood samples were removed from the packs, placed on ice, and processed immediately. While the baboons were sedated, a sample was collected by venipuncture to measure cortisol, a substance that changes slowly in response to events. Typically, samples were collected twice a week. A complete round of data collection from one pair of males included repeated trials during anticipation and presentation of preferred food, quiet times, and exposure to an intruder. The collection process took about 2 months.

An example of data collected with this system is presented in Figure 4. The data show an animal's heart rate 

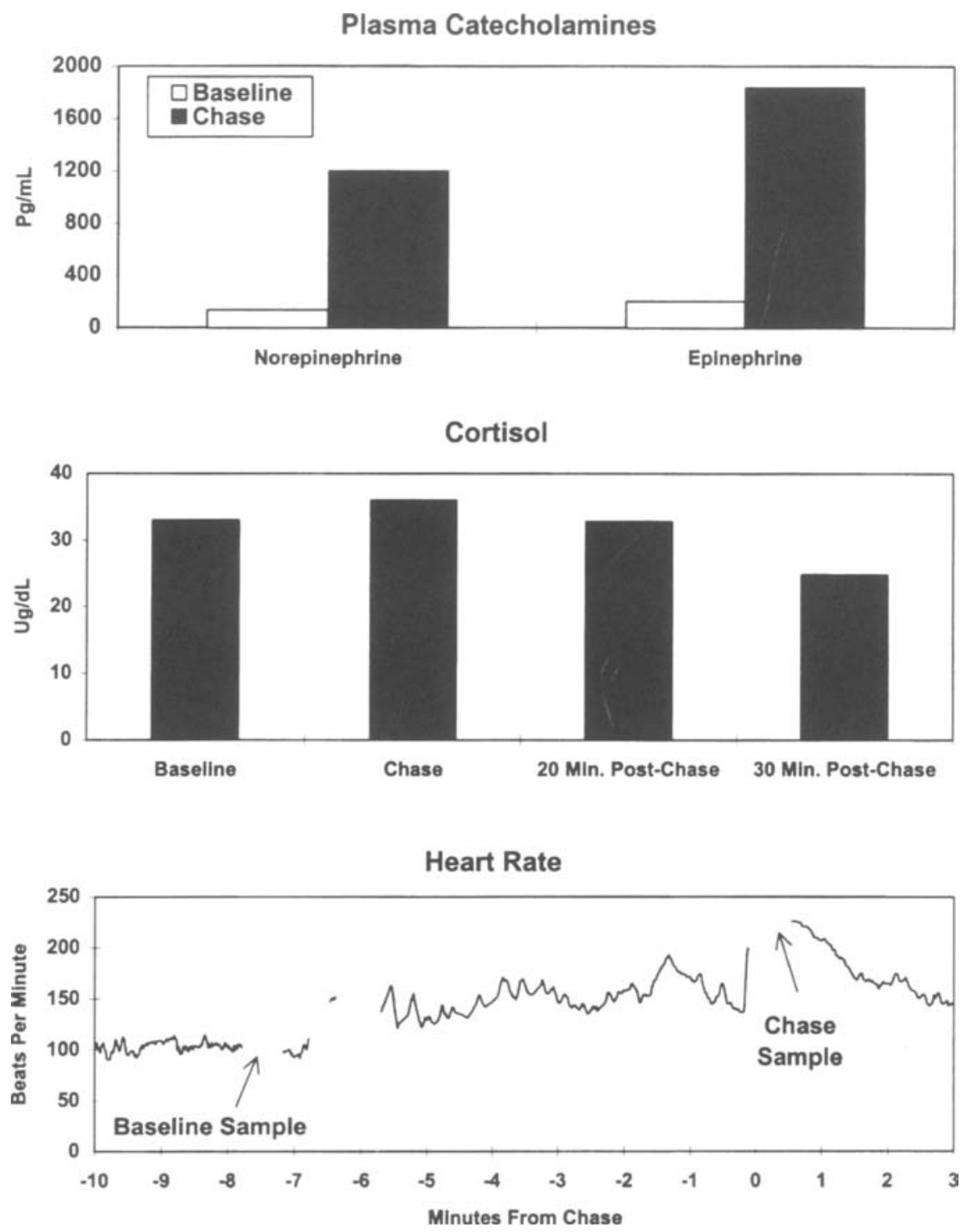

Figure 4. Physiological responses to a chase.

and plasma catecholamine and cortisol levels during a food anticipation/presentation session in which the animal became involved in a chase. Levels designated as baseline were from a sample collected when the animal had been sitting calmly for $4 \mathrm{~min}$. Heart rate before the chase was influenced by preparation and presentation of food beginning $7 \mathrm{~min}$ before the chase occurred. About $90 \mathrm{sec}$ before the chase, the subject spent $4 \mathrm{sec}$ directing aggression toward the other male, which was eating some of the food. The other male did not return aggression at that point. Heart rate increased sharply just before the baboons actually began chasing each other. Sample collection began $10 \mathrm{sec}$ after the onset of the $21-\mathrm{sec}$ chase and lasted $30 \mathrm{sec}$.

Plasma norepinephrine and epinephrine levels were about nine times higher in the chase sample than they were in the baseline. The catecholamine levels far exceeded levels typically seen at that time point in the food anticipation and presentation paradigm (norepinephrine, $1,200 \mathrm{pg} / \mathrm{ml}$ vs. $400 \mathrm{pg} / \mathrm{ml}$; epinephrine, $1,837 \mathrm{pg} / \mathrm{ml}$ vs. $200 \mathrm{pg} / \mathrm{ml}$ ). Cortisol levels in samples collected after the baboon was sedated with Telazol (tiletamine and zolazepam) were not higher than levels seen during the chase. The baseline cortisol level was unusually high for captive baboons but was not unusual for this subject when it was a member of a group in which dominance was contested.

Plasma catecholamines degrade in whole blood and in plasma at room temperature. These steps were taken to minimize the degradation and to test for its occurrence: (1) Sodium metabisulfite $(5 \mathrm{mg})$ was added to each blood collection tube. (2) The baboons were trained to 
leave the compound and enter an adjacent holding area quickly so samples could be retrieved expeditiously. (3) Intraarterial delivery of ketamine by remote control minimized the time it took to immobilize the baboons. (4) As soon as baboons were immobilized, blood samples were placed on ice and centrifuged. Plasma was stored at $-70^{\circ} \mathrm{C}$. (5) Catecholamine levels in baseline samples collected by remote control up to $45 \mathrm{~min}$ apart did not differ significantly from each other.

\section{DISCUSSION}

We collected more than 200 samples by remote control from 6 baboons. We rarely needed to reschedule collections because of problems with the device, and, in the last pair of baboons studied, $91 \%$ of sampling attempts were successful.

Before the devices performed reliably, two major challenges had to be addressed: (1) maintaining patency of the catheter for several weeks while not jeopardizing the subjects' health by overinjection of heparin, and (2) preventing vacuum loss in the collection tubes. In the 2 years the device has been in use, no catheters have been lost because of permanent occlusion. In the beginning, however, clots formed in the catheter, requiring sedation of the baboons and posing a risk of releasing a clot to the animal's bloodstream. (The catheter was situated so that if a clot was passed, it would enter the arterial tree below the brain, heart, and lungs.) The problem was solved by elimination of all points where the animal's arterial pressure could push heparin out of the catheter. Plastic connectors and plugs were used in place of stopcocks, and the rocker isolation valves were pretested to ensure that fluid could not pass through them when they were in the closed state. Finally, each new device was tested by overnight application of $200-\mathrm{mm} \mathrm{Hg}$ air pressure. In addition to leak prevention, long-term catheter maintenance included remote-controlled injection of $0.1-0.4 \mathrm{ml}$ of heparin twice a day to keep the end of the catheter open and remove any blood that may have advanced into it. Urokinase was used once a month to degrade fibrinogen and other substances that may have built up on the inner surface of the catheter. Each time the baboons were sedated, a saline-filled syringe was used to gently advance and withdraw fluid through the catheter tip. Blood was thoroughly flushed from the catheter with saline, which was then replaced with heparin.

Visual feedback from the blood pressure signal was essential to maintaining the catheter and ensuring that blood was removed from it between samples. The amplitude and shape of the signal indicated when a catheter was sufficiently cleared of blood. Occasionally, a leak developed while the subject was wearing a pack. This was detected quickly by examining the blood pressure signal during daily catheter maintenance. When a leak was detected, a backup unit was used while the leak was located and eliminated. Following blood collection, release of heparin from the long-duration syringe typically did not completely fill the dead space of the catheter $(\sim 1.2 \mathrm{ml})$, so its release was followed by injections from the short-duration syringe just until the blood pressure signal regained its correct appearance. This prevented release of excessive amounts of heparin into the subjects. The amount of heparin injected during routine maintenance and sample collection was not observed to impair clot formation, affect blood pressure or hematocrit over time, or otherwise impact the health of the baboons.

A few early samples were unusable owing to loss of vacuum in the collection tubes. The tubes had been vacuumsealed before being loaded into the pack, and the jostling that occurs during loading may have produced brief gaps between the tubing and adjacent surfaces or between the needle and stopper, leading to loss of the seal. By applying the vacuum only after the device has been loaded into the pack, we obtained a dramatic improvement in vacuum retention; samples are rarely lost to poor vacuum seals.

The device made it possible to determine cortisol and plasma catecholamine levels at the same time cardiovascular and behavioral data were recorded in dominant and subordinate baboons before and during events. The baboons were not aware that samples were being collected, and their routines were not disrupted before the event, so data reflect true baseline conditions and responses to the events themselves. Because the device was designed for use on a long-term basis, we were able to obtain repeated measures in the same baboon during various events. This made it possible to study the animal's physiological responses during a wide variety of behaviors and social responses in a free-ranging environment. The ability to unobtrusively collect samples at any time made it possible to determine event-related plasma catecholamine levels, which can change rapidly, at particular points.

The device can be used to study baseline and midevent levels of many other rapidly fluctuating bloodborne substances, including ones that are influenced by psychological or psychosocial stimuli. One valuable application of the device would be to study the time kinetics of changes in levels of many blood-borne substances in response to psychological events with a well-defined onset. Such information may help resolve conflicting results obtained by different investigators studying the same phenomenon but collecting samples at different points in time (Richter et al., 1996). If the device were adapted for use in human studies, it would have the added advantage of minimizing placebo effects. The investigator could collect a baseline sample, inject an agent, and then collect later samples without the subject's awareness of when the agent enters the body or when samples are drawn. The person could be free to move about a room and engage in various activities throughout data collection.

\section{Availability}

This system is not commercially available. Some aspects of the design need to be specifically tailored to the species, setting, and goals of the investigator. For details 
about remote-controlled blood draws and making collection/injection units, contact K. L. Bentson. Contact the Washington Regional Primate Research Center (WRPRC) Bioengineering Department for information about obtaining schematics, software, mechanical drawings, circuit board data files, and basic construction procedures concerning radio telemetry, the controller card, and the command link. The WRPRC may also be contacted about having components of the system built for the investigator.

\section{REFERENCES}

Astley, C. A., Smith, O. A., Ray, R. D., Golanov, E. V., Chesney, M. A., Chalyan, V. G., Taylor, D. J., \& Bowden, D. M. (1991). Integrating behavior and cardiovascular responses: The code. American Journal of Physiology, 261, R172-R181.

BENTSON, K. L. (1998). Hormonal, cardiovascular, and behavioral correlates of rank in male baboons during activities that occur in a social setting. Dissertation Abstracts International, 58, 9819206.

Bentson, K. L., Astley, C. A., Miles, F. P., Goldstein, D. S., Holmes, C., \& Smith, O. A. (1995), Cardiovascular and neuroendocrine responses to chases and food anticipation in baboons: A study involving telemetry and remote control blood draws [Abstract]. American Journal of Primatology, 36, 109.

Bentson, K. L., Astley, C. A., Miles, F. P., Goldstein, D. S., Holmes, C., \& Smith, O. A. (1996). Behavioral and physiological aspects of exposure of a group of baboons to an intruder [Abstract]. In Program for August 1996 Meeting of the International Primatological Society and the American Society of Primatologists.
Bentson, K. L., Astley, C. A., Miles, F. P., Goldstein, D. S., Holmes, C., \& SMith, O. A. (1997). Rank differences and similarities in physiological responses to food anticipation and presentation in free-ranging captive baboons [Abstract]. American Journal of Primatology, 42, 94-95.

Herndon, J. G., Perachio, A. A., Turner, J. J., \& Collins, D. C. (1981). Fluctuations in testosterone levels of male rhesus monkeys during copulatory activity. Physiology \& Behavior, 26, 525-528.

HILL, R. D. (1986). Microcomputer monitor and blood sampler for freediving Weddell seals. Journal of Applied Physiology, 61, 1570-1576.

Morton, W. R., Knitter, G. H., Smith, P. M., Susor, T. G., \& Schmiтt, K. (1987). Alternatives to chronic restraint of nonhuman primates. Journal of the American Veterinary Association, 191, 12821286.

Richter, S. D., Schurmeyer, H., Schedlowski, M., Hadicke, A., Tewes, U., Schmidt, R. E., \& Wagner, T. O. F. (1996). Time kinetics of the endocrine response to acute psychological stress. Journal of Clinical Endocrinology \& Metabolism, 81, 1956-1960.

Scheid, P.. \& Slama, H. (1975). Remote-controlled device for sampling arterial blood in unrestrained animals. Pflügers Archiv, 356, 373-376.

Schmalzried, R. T., Toll, P. W., Devore, J. J., \& Fedde, M. R. (1992). Microcontroller-based system for collecting anaerobic blood samples from a running greyhound. Computer Methods \& Programs in Biomedicine, 37, 183-190.

Spelman, F. A., Astley, C. A., Golanov, E. V., Cupal, J., Henkins, A. R., Fonzo, E., Susor, T. G., McMorrow, G., Bowden, D. M., \& Smith, O. A. (1991). A system to acquire and record physiological and behavioral data remotely from nonhuman primates. IEEE Transactions on Biomedical Engineering, 38, 1175-1185.

(Manuscript received May 11, 1998; revision accepted for publication August 20, 1998.) 